\title{
Many-Body Chaos in the Sachdev-Ye-Kitaev Model
}

\author{
Bryce Kobrin, ${ }^{1,2}$ Zhenbin Yang, ${ }^{3,4}$ Gregory D. Kahanamoku-Meyer®, ${ }^{1}$ Christopher T. Olund®, ${ }^{1}$ \\ Joel E. Moore, ${ }^{1,2}$ Douglas Stanford, ${ }^{4,5}$ and Norman Y. Yao $\circledast^{1,2}$ \\ ${ }^{1}$ Department of Physics, University of California, Berkeley, California 94720, USA \\ ${ }^{2}$ Materials Sciences Division, Lawrence Berkeley National Laboratory, Berkeley, California 94720, USA \\ ${ }^{3}$ Department of Physics, Princeton University, Princeton, New Jersey 08540, USA \\ ${ }^{4}$ Stanford Institute for Theoretical Physics, Stanford, California 94305, USA \\ ${ }^{5}$ Institute for Advanced Study, Princeton, New Jersey 08540, USA
}

(Received 24 April 2020; revised 6 October 2020; accepted 17 November 2020; published 20 January 2021)

\begin{abstract}
Many-body chaos has emerged as a powerful framework for understanding thermalization in strongly interacting quantum systems. While recent analytic advances have sharpened our intuition for many-body chaos in certain large $N$ theories, it has proven challenging to develop precise numerical tools capable of exploring this phenomenon in generic Hamiltonians. To this end, we utilize massively parallel, matrix-free Krylov subspace methods to calculate dynamical correlators in the Sachdev-Ye-Kitaev model for up to $N=60$ Majorana fermions. We begin by showing that numerical results for two-point correlation functions agree at high temperatures with dynamical mean field solutions, while at low temperatures finite-size corrections are quantitatively reproduced by the exactly solvable dynamics of near extremal black holes. Motivated by these results, we develop a novel finite-size rescaling procedure for analyzing the growth of outof-time-order correlators. Our procedure accurately determines the Lyapunov exponent, $\lambda$, across a wide range in temperatures, including in the regime where $\lambda$ approaches the universal bound, $\lambda=2 \pi / \beta$.
\end{abstract}

DOI: 10.1103/PhysRevLett.126.030602

Characterizing thermalization in strongly interacting quantum systems is a goal that spans across multiple disciplines ranging from condensed matter and quantum information to quantum gravity. Recent developments toward this goal have revealed striking insights into the relationship between quantum chaos and the delocalization, or scrambling, of quantum information. This unification is partly provided by the notion of out-of-time-order correlators (OTOCs), which take the general form $\langle W(t) V(0) W(t) V(0)\rangle$ for local operators $V$ and $W$ [1-3]. From an information theoretic perspective, these correlators determine the degree to which local information becomes hidden in nonlocal degrees of freedom, leading to the effective memory loss of initial conditions $[2,4]$. From the perspective of chaos, OTOCs measure the sensitivity of one operator toward a small perturbation induced by another operator at an earlier time $[5,6]$. In particular, for semiclassical chaotic systems, OTOCs are expected to exhibit a period of exponential growth analogous to the classical butterfly effect $[7,8]$.

At the intersection between these two perspectives lies the discovery of a new form of quantum chaos in strongly

Published by the American Physical Society under the terms of the Creative Commons Attribution 4.0 International license. Further distribution of this work must maintain attribution to the author(s) and the published article's title, journal citation, and DOI. Funded by SCOAP . interacting systems, known as many-body chaos. This phenomenon is characterized by OTOCs whose leading order behavior is given by $e^{\lambda t} / N$, where $\lambda$ is the Lyapunov exponent and $N$ is related to the number of degrees of freedom per site [5,9]. While such behavior was first anticipated in [9] and confirmed using holographic duality in [2], the first concrete Hamiltonian model to exhibit
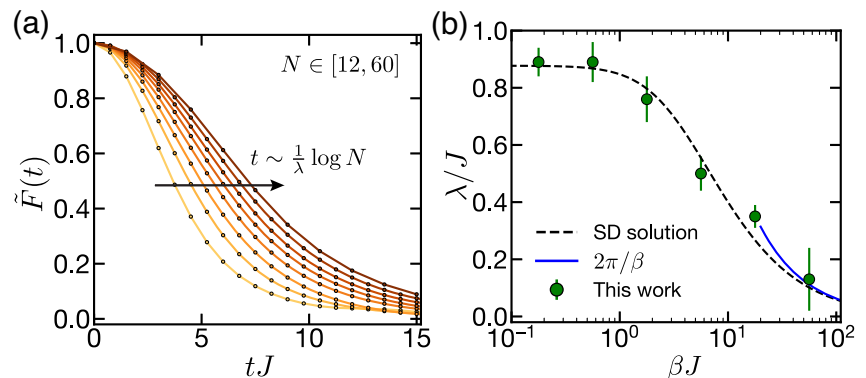

FIG. 1. Regularized OTOCs in the SYK model, $\tilde{F}(t) \equiv F(t) / F(0)$, as shown for $\beta J=10$ and system sizes $N \in[12,60]$. The early time behavior is characterized by $1-$ $\tilde{F}(t) \sim e^{\lambda t} / N$ and different system sizes are approximately related by a time translation symmetry, $t \rightarrow t+1 / \lambda \log N$. (b) Applying a finite-size rescaling procedure to the data, we determine $\lambda$ as a function of temperature (points). Our results exhibit excellent agreement with the theoretical predictions of the SchwingerDyson (SD) equations (dashed line), including in the regime where $\lambda$ approaches the bound on chaos $2 \pi / \beta$ (blue). 
many-body chaos was introduced by Kitaev following previous work by Sachdev and Ye [10-13]. Remarkably, at low temperatures, the Lyapunov exponent of this so-called Sachdev-Ye-Kitaev (SYK) model saturates a universal bound, $\lambda \leq 2 \pi T$, where $T$ is the temperature of the system [5]. The saturation of this bound is known to occur in theories of quantum gravity and their holographic duals [6], and indeed a direct correspondence has since been established between the low temperature dynamics of the SYK model and a universal theory of near extremal black holes (i.e., Jackiw-Teitelboim gravity) [13-16]. More recently, a number of other models that exhibit many-body chaos have been studied; however, their rate of chaos is parametrically slower than the thermodynamic bound $[17,18]$. In parallel, there have also been numerous proposals to directly measure OTOCs in coherently controlled quantum simulators [8,19-27], as well as a number of experimental demonstrations in small-scale systems [24,28-30].

A major hurdle in benchmarking these experiments/ proposals and in identifying novel models that exhibit many-body chaos is the lack of a reliable numerical toolset. Indeed, in order to observe a period of clear exponential growth, the scrambling time must be well separated from other effects related to local relaxation that occur at early times [5,21,31].

In this Letter, we take steps to overcome these challenges by employing massively parallelized Krylov subspace methods and developing new extrapolation tools to characterize many-body chaos. Specifically, we compute correlation functions for the SYK model for systems of up to $N=60$ Majorana fermions and leverage the model's correspondence with quantum gravity to interpret finitesize effects. We present two main results. First, we demonstrate that our numerical results for two-point functions, $G(t)=\langle W(t) W(0)\rangle$, agree quantitatively with analytic predictions in two distinct regimes: (i) at high temperatures, our results match the mean-field solution of the microscopic model, and (ii) at low temperatures, our results are consistent with the full quantum dynamics of near extremal black holes. These latter results represent, to the best of our knowledge, the first direct numerical verification of quantum gravity correlators and highlight the close connection between finitesize corrections and gravitational fluctuations.

Second, we introduce an extrapolation procedure for determining the Lyapunov exponent that explicitly takes into account higher-order terms in the OTOCs. We verify that this procedure accurately determines $\lambda$ as a function of temperature, including at low temperatures where $\lambda \approx 2 \pi T$ (Fig. 1).

The SYK model and its gravity dual.-Consider the SYK Hamiltonian given by $[11,12]$

$$
H=\frac{6}{N^{3}} \sum_{i<j<k<l} J_{i j k l} \chi_{i} \chi_{j} \chi_{k} \chi_{l} .
$$

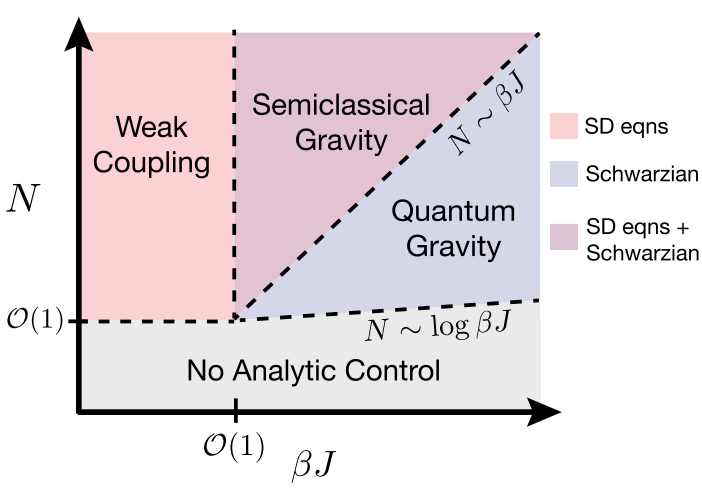

FIG. 2. Regimes of analytic control for the SYK model as a function of system size, $N$, and inverse temperature, $\beta J$. In the semiclassical limit (red and purple), the model is well described by a dynamical mean-field solution (Schwinger-Dyson equations). At low temperatures, finite-size corrections can be calculated using the Schwarzian action (blue), which is dual to two-dimensional antide Sitter gravity. However, at sufficiently small sizes (gray), the dynamics are governed by the discreteness of the energy spectrum and neither effective theory provides a valid description.

Here $\chi_{i}(i=1, \ldots, N)$ are Majorana fermions which obey the anticommutation relation, $\left\{\chi_{i}, \chi_{j}\right\}=\delta_{i j}$, and $J_{i j k l}$ are random (real) coefficients sampled from a Gaussian distribution characterized by a standard deviation $\sigma=J$.

In order to probe the system's nonequilibrium dynamics, we will compute two different types of correlators. In-time correlators reveal how excitations in the system relax toward equilibrium. In particular, we will consider the average imaginary-time Green's function, $G(\tau)$, and its real-time cousin, $G_{R}(t)$, given by

$$
\begin{aligned}
& G(\tau) \equiv{\overline{\left\langle\chi_{i}(\tau) \chi_{i}(0)\right\rangle_{\beta}}} \\
& G_{R}(t) \equiv 2 \operatorname{Re}\left[\overline{\left\langle\chi_{i}(t) \chi_{i}(0)\right\rangle_{\beta}}\right],
\end{aligned}
$$

where $\tau(t)>0$ is imaginary (real) time, $\langle\cdots\rangle_{\beta}=$ $1 / Z \operatorname{Tr}\left[\cdots e^{-\beta H}\right]$ is a thermal average at inverse temperature $\beta=1 / T$, and the overline denotes the (quenched) average over disorder realizations. On the other hand, to probe chaos and the scrambling of quantum information, we will consider out-of-time-order correlators. We will primarily focus on the regularized OTOC,

$$
F^{(r)}(t) \equiv \overline{\left\langle\chi_{i}(t) \rho^{\frac{1}{4}} \chi_{j}(0) \rho^{\frac{1}{4}} \chi_{i}(t) \rho^{\frac{1}{4}} \chi_{j}(0) \rho^{\frac{1}{4}}\right\rangle}
$$

where $i \neq j$, and $\rho=e^{-\beta H}$, the imaginary-time evolution associated with the thermal ensemble, is distributed evenly among the four operators. In the Supplemental Material, we provide a detailed discussion regarding the key differences between this correlator and the unregularized version $[32,33]$.

In the large $N$, semiclassical limit, both in-time and outof-time correlators can be exactly computed via a diagrammatic approach $[11,12]$. The average Green's functions 
are determined by the self-consistent Schwinger-Dyson equations. For the OTOCs, the leading order term in $1 / N$ is computed by summing a series of ladder diagrams.

Beyond the semiclassical limit, the dynamics at low temperature (i.e., $\beta J \gg 1$ ) are captured by an effective theory known as the "Schwarzian theory" (Fig. 2) [11,13,43,44]. The same theory also describes Jackiw-Teitelboim gravity, a simple quantum gravity description of two-dimensional anti-de Sitter space.

Crucially, correlators in the Schwarzian theory are exactly computable [44,45], which will enable us to perform quantitative, finite-size-scaling comparisons for two-point functions $G(\tau)$ and $G_{R}(t)$ outside of the semiclassical limit. However, for the four-point function, the expressions are more complicated, and we will compare numerics to the ansatz: $F(t)=C_{0}+C_{1}\left(e^{\lambda t} / N\right)+C_{2}\left(e^{\lambda t} / N\right)^{2}+\cdots$, which is valid for large $N$ and $t \lesssim 1 / \lambda \log N[15,44,45]$. An analogous series expansion is expected to characterize OTOCs for the SYK model at high temperatures (and any other model described by ladder diagrams) $[17,41,46]$.

Nonequilibrium dynamics in the SYK model.-Our central numerical tool is a massively parallelized implementation of a class of iterative methods known as Krylov subspace methods $[33,47,48]$. These methods approximate the time evolution of an initial state, $|\psi(t)\rangle=e^{-i H t}|\psi\rangle$, within a subspace formed by successive applications of the Hamiltonian. Since this requires an initial pure state, we approximate thermal averages by taking the expectation value with respect to a Haar-random state $|\tilde{\psi}\rangle$ [49-51],

$$
\operatorname{Tr}\left[\hat{O} e^{-\beta H}\right] \approx\left\langle\tilde{\psi}\left|e^{-\frac{\beta}{2} H} \hat{O} e^{-\frac{\beta}{2} H}\right| \tilde{\psi}\right\rangle .
$$

Owing to quantum typicality, the error in this approximation scales inversely with the number of states in the thermal ensemble and, thus, decreases exponentially with $N$ for arbitrary systems and temperatures (above the spectral gap) [50]. In practice, we further reduce the error by averaging over initial states [33].

To begin probing the thermalizing dynamics of the SYK model, we compute the average Green's functions for both real- and imaginary-time evolution in the temperature range, $0<\beta J \leq 100$. At high temperatures, the imaginary-time Green's function, $G(\tau)$, shows excellent agreement with the semiclassical solution given by the Schwinger-Dyson equations [Fig. 3(a)]. At lower temperatures, the difference between our numerics and the semiclassical solutions widens. To understand the origin of these corrections, we plot the full solution predicted by the Schwarzian action. This exhibits close quantitative agreement with our data at temperatures corresponding to $\beta J \gtrsim 50$. Crucially, this confirms that the Schwarzian action, or its corresponding gravity dual, accurately captures finite-size corrections away from the semiclassical regime.

A few remarks are in order. First, we note that the agreement with the Schwarzian action is only valid for
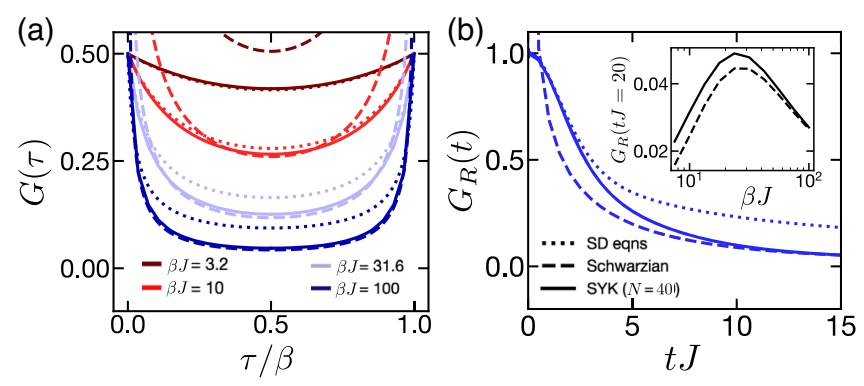

FIG. 3. Two-point correlation functions in real and imaginary time. (a) Comparison of imaginary-time evolution between our numerics with 40 Majoranas (solid), the large $N$ solution (dotted), and the Schwarzian action (dashed). At high temperatures, we observe quantitative agreement between our numerical results and the large $N$ solution, while at low temperatures our numerics are well described by the Schwarzian action. (b) Analogous comparison for real-time evolution with $\beta J=56$. Our numerics show excellent agreement with the Schwarzian action for $t J \gtrsim 10$. The disagreement at earlier times is attributed to the difference in highenergy modes, which are cut off at the energy scale $J$ in the SYK model and are unbounded in the effective action. Inset: a salient feature in our real-time numerics is a nonmonotonic trend with respect to temperature, as shown for $t J=20$. This behavior is captured by the Schwarzian action (dashed) and can be understood as a consequence of the square root edge of the energy spectrum.

system sizes larger than $N \approx 30$ [33]. For smaller sizes, we observe additional finite-size corrections that are attributed to the discreteness of the energy spectrum. Such nonSchwarzian corrections are expected to dominate when the temperature approaches the energy of the level spacings (i.e., $N \sim \log \beta J$ in Fig. 2) [52,53]. Second, the agreement between the Schwarzian and our numerics does not hold at time scales shorter than the inverse of the microscopic coupling strength (i.e., $\tau J \lesssim 1$ ); specifically, the Schwarzian dynamics diverge as $\tau J \rightarrow 0$ while our numerics approach a finite value. This difference arises from the fact that the Schwarzian action is the effective theory only at low energies (compared to $J$ ); for higher energies, the SYK dynamics are governed by the microscopic nature of the model.

Much like the imaginary-time case, we find that the retarded Green's function, $G_{R}(t)$, agrees with the semiclassical solutions at high temperatures and with the full dynamics of the Schwarzian action at low temperature [Fig. 3(b)]. We note, however, that the early time discrepancy with the Schwarzian action is extended to later times (i.e., $\beta J \sim 10$ ). This can be attributed to the longer time scale required for the phase cancellation of the high-energy modes in real time, as opposed to the direct suppression that occurs in imaginary time.

Working with real-time dynamics also allows us to probe a rather nontrivial prediction of the Schwarzian action. In particular, one expects the late-time dynamics to be governed by the functional form of the spectral density at low energies, $\rho(E) \sim E^{(1 / 2)}[43,54,55]$. This square-root 
singularity leads to a power-law decay of the Green's function, with a power that depends on both the temperature and the time scale. Intriguingly, it predicts a nonmonotonic temperature dependence for the decay of the Green's function, in stark contrast to the monotonic dependence predicted by the semiclassical solution. This nontrivial temperature dependence, consistent with only the full Schwarzian solution, is indeed borne out by the numerics [inset, Fig. 3(b)].

Lyapunov exponent of the SYK model.-To probe manybody chaos in the SYK model, we now compute regularized OTOCs [Eq. (4)] for temperatures in the range $0<\beta J \leq 56$ and for system sizes up to $N=60$. In the large $N$ limit, one expects a well-defined period of exponential growth, starting from the time scale at which the two-point functions decay and persisting until the scrambling time [5]. However, for conventional exact diagonalization studies, there is little separation between these time scales, owing to the limited system sizes that are numerically accessible; indeed, prior studies actually observed an increase in the extracted Lyapunov exponent as a function of decreasing temperature-the opposite behavior of what is expected $[33,56]$. By scaling to larger system sizes using Krylov subspace methods, we observe a direct turnover in this trend. Moreover, we introduce a novel extrapolation method, which provides a robust way of extracting the Lyapunov exponent.

The intuition behind our method is as follows: for a large class of many-body chaotic systems, the full form of the OTOC in the semiclassical limit is given by a series in $e^{\lambda t} / N$. Crucially, this series exhibits a rescaling symmetry, wherein $N \rightarrow r N$ amounts to shifting the full curve by $t \rightarrow t+1 / \lambda \log r$. This symmetry can be shown explicitly for the Schwarzian action, which governs low-temperature dynamics of the SYK model, and is also expected to hold at high temperatures $[17,41]$.

This suggests that we can determine $\lambda$ at a given temperature by attempting to collapse our data [Fig. 1(a)] through finite-size rescaling of the form $t \rightarrow t+1 / \lambda \log N$. More specifically, we first interpolate our data to find the time, $t_{*}$, at which each curve crosses a fixed value, i.e., $F\left(t_{*}\right) / F(0)=1-F_{0}$ [33]. Next, we estimate $\lambda_{\text {fit }}(N)$ as $1 / \lambda_{\text {fit }}=d t_{*} / d(\log N)$, where $N$ corresponds to the system size about which we take the numerical derivative. Finally, we fit our results to a $1 / N$ series, $\lambda_{\text {fit }}(N)=$ $\lambda_{0}+\lambda_{1} / N+\lambda_{2} / N^{2}+\cdots$; the leading order term $\lambda_{0}$ corresponds to the extrapolated value for $\lambda$ as $N \rightarrow \infty$.

In Fig. 1(b), we present our results for $\lambda_{0}$ as a function of temperature. We observe excellent agreement with analytic predictions for all temperatures in the range $0<\beta J \leq 56$. Crucially, our protocol works at low temperatures where the $2 \pi / \beta$ scaling (saturating the bound on chaos) becomes apparent.

An important question to ask is over what range of temperatures we expect our procedure to remain valid.
There are three relevant considerations. First, the temperature must be high compared to the energy associated with the level spacing; we account for this requirement by considering only system sizes where at least 20 eigenstates, on average, lie within $\Delta E=1 / \beta$ of the ground state. Second, the system must be sufficiently close to the semiclassical limit for the rescaling symmetry to hold. It is known from the Schwarzian action that this condition corresponds to $\beta J \lesssim N$. Asymptotically, this is a much stronger requirement than the first condition; however, for the system sizes relevant for our study $(N \lesssim 60)$, both requirements imply a low temperature limit of $\beta J \approx 60$.

Third, there must sufficient separation between the scrambling time and the short-time dissipative dynamics. In the case of the regularized correlator, this condition is given by $\beta J \lesssim N$, leading to the same temperature range as the semiclassical requirement. However, in the case of unregularized correlators, the corresponding condition is $(\beta J)^{3} \lesssim N$; this implies that the unregularized correlator is subject to stronger finite-size effects, which we corroborate through our numerics [33].

Discussion and outlook.-By employing massively parallelized Krylov subspace methods and developing novel extrapolation tools, we have demonstrated that one can utilize numerics to accurately capture the thermalizing and chaotic dynamics of the SYK model. Our results for two-point Green's functions represent a direct verification of the dynamics of quantum black holes in a highly fluctuating regime. Moreover, our finite-size rescaling procedure for extracting Lyapunov exponents leads to the first numerical evidence that the SYK model saturates the theoretical bound on chaos, $\lambda \approx 2 \pi T$.

We anticipate that the numerical tools demonstrated here will open the door to a number of intriguing future directions. First, our numerical tools can be applied to variations of the SYK model (i.e., large $q$ limit) for which the effective action (i.e., Liouville action) is known for all temperatures $[55,57,58]$. This will enable quantitative studies of finite-size corrections in the high-temperature regime, where the Schwarzian action is not valid. Second, our procedure for characterizing Lyapunov exponents can diagnose many-body chaos in other models beyond the SYK model; this is of particular relevance for experimental platforms which have constraints on the types of interactions and disorder that can be realized $[26,29,30,59,60]$. Finally, we envision future numerical simulations to test more complex gravitational phenomena, including traversable wormholes [61,62], and the possible emergence of SYK dynamics in transport experiments of quantum materials [63-65].

We gratefully acknowledge the insights of and discussions with Felix Flicker, Snir Gazit, Thomas Scaffidi, Pratik Rath, Markus Schmitt, Nicole Yunger Halpern, Brian Swingle, Victor Galitski, Stephan Plugge, and Beni Yoshida. This work was supported by the U.S. 
Department of Energy through the Quantum Information Science Enabled Discovery (QuantISED) for High Energy Physics (KA2401032) and through the GeoFlow Grant No. de-sc0019380. This research used resources of the National Energy Research Scientific Computing Center, a U.S. Department of Energy Office of Science User Facility operated under Contract No. DE-AC02-05CH11231. The numerical work performed in this work used the DYNAMITE Python front end [48], which supports a matrix-free implementation of Krylov subspace methods based on the PETSC and SLEPC packages [38-40]. J. E. M. acknowledges support of NSF DMR-1918065 and a Simons Investigatorship. G. D. K.-M. acknowledges support by the Department of Defense through the National Defense Science and Engineering Graduate Fellowship Program. C. T. O. acknowledges support by the Department of Defense through the National Defense Science and Engineering Graduate Fellowship program.

[1] A. I. Larkin and Y. N. Ovchinnikov, Sov Phys JETP 28, 1200 (1969).

[2] S. H. Shenker and D. Stanford, J. High Energy Phys. 03 (2014) 067.

[3] A. Kitaev, Fundamental Physics Prize Symposium (2014), https://www.youtube.com/watch?v=OQ9qN8j7EZI.

[4] P. Hosur, X.-L. Qi, D. A. Roberts, and B. Yoshida, J. High Energy Phys. 02 (2016) 004.

[5] J. Maldacena, S. H. Shenker, and D. Stanford, J. High Energy Phys. 08 (2016) 106.

[6] S. H. Shenker and D. Stanford, J. High Energy Phys. 05 (2015) 132.

[7] Y. Liao and V. Galitski, Phys. Rev. B 98, 205124 (2018).

[8] R. J. Lewis-Swan, A. Safavi-Naini, J. J. Bollinger, and A. M. Rey, Nat. Commun. 10, 1581 (2019).

[9] Y. Sekino and L. Susskind, J. High Energy Phys. 10 (2008) 065.

[10] S. Sachdev and J. Ye, Phys. Rev. Lett. 70, 3339 (1993).

[11] J. Maldacena and D. Stanford, Phys. Rev. D 94, 106002 (2016).

[12] A. Kitaev, A Simple Model of Quantum Holography (2015), http://online.kitp.ucsb.edu/online/entangled15/kitaev/, http://online.kitp.ucsb.edu/online/entangled15/kitaev2/.

[13] A. Kitaev and S. J. Suh, J. High Energy Phys. 05 (2018) 183.

[14] K. Jensen, Phys. Rev. Lett. 117, 111601 (2016).

[15] J. Maldacena, D. Stanford, and Z. Yang, arXiv:1606.01857.

[16] J. Engelsöy, T. G. Mertens, and H. Verlinde, J. High Energy Phys. 07 (2016) 139.

[17] D. Stanford, J. High Energy Phys. 10 (2016) 009.

[18] D. Chowdhury and B. Swingle, Phys. Rev. D 96, 065005 (2017).

[19] M. K. Joshi, A. Elben, B. Vermersch, T. Brydges, C. Maier, P. Zoller, R. Blatt, and C. F. Roos, Phys. Rev. Lett. 124, 240505 (2020).

[20] É. Lantagne-Hurtubise, S. Plugge, O. Can, and M. Franz, Phys. Rev. Research 2, 013254 (2020).
[21] N. Y. Yao, F. Grusdt, B. Swingle, M. D. Lukin, D. M. Stamper-Kurn, J.E. Moore, and E. A. Demler, arXiv:1607.01801.

[22] G. Zhu, M. Hafezi, and T. Grover, Phys. Rev. A 94, 062329 (2016).

[23] B. Swingle, G. Bentsen, M. Schleier-Smith, and P. Hayden, Phys. Rev. A 94, 040302(R) (2016).

[24] J. Li, R. Fan, H. Wang, B. Ye, B. Zeng, H. Zhai, X. Peng, and J. Du, Phys. Rev. X 7, 031011 (2017).

[25] J. Dressel, J. R. Gonzalez Alonso, M. Waegell, and N. Halpern, Phys. Rev. A 98, 012132 (2018).

[26] G. Bentsen, T. Hashizume, A. S. Buyskikh, E. J. Davis, A. J. Daley, S. S. Gubser, and M. Schleier-Smith, Phys. Rev. Lett. 123, 130601 (2019).

[27] B. Yoshida and N. Y. Yao, Phys. Rev. X 9, 011006 (2019).

[28] M. Gärttner, J. G. Bohnet, A. Safavi-Naini, M. L. Wall, J. J. Bollinger, and A. M. Rey, Nat. Phys. 13, 781 (2017).

[29] K. A. Landsman, C. Figgatt, T. Schuster, N. M. Linke, B. Yoshida, N. Y. Yao, and C. Monroe, Nature (London) 567, 61 (2019).

[30] M. Blok, V. Ramasesh, T. Schuster, K. O’Brien, J. Kreikebaum, D. Dahlen, A. Morvan, B. Yoshida, N. Yao, and I. Siddiqi, arXiv:2003.03307.

[31] D. Bagrets, A. Altland, and A. Kamenev, Nucl. Phys. B921, 727 (2017).

[32] Of course, the unregularized OTOC, $F^{(u)}(t)=$ $\langle W(t) V(0) W(t) V(0) \rho\rangle$, is often more relevant for experiments and we provide a detailed discussion of the differences in the Supplemental Material [33]. In particular, we discuss the importance of the magnitude $C_{1}$ of the leading exponentially growing term in the OTOC, $\left(C_{1} / N\right) e^{\lambda t}$ [41]. This magnitude determines the separation between the scrambling time and the early time, dissipative dynamics, and crucially varies significantly between the two types of OTOCs (regularized vs unregularized) [7,20,42].

[33] See Supplemental Material at http://link.aps.org/ supplemental/10.1103/PhysRevLett.126.030602 for additional numerics, derivations, and analysis, which includes Refs. [34-40].

[34] H. Shen, P. Zhang, R. Fan, and H. Zhai, Phys. Rev. B 96, 054503 (2017).

[35] A. Keleş, E. Zhao, and W. V. Liu, Phys. Rev. A 99, 053620 (2019).

[36] O. Parcollet and A. Georges, Phys. Rev. B 59, 5341 (1999).

[37] A. Eberlein, V. Kasper, S. Sachdev, and J. Steinberg, Phys. Rev. B 96, 205123 (2017).

[38] V. Hernandez, J. E. Roman, and V. Vidal, ACM Trans. Math. Softw. 31, 351 (2005).

[39] J. E. Roman, C. Campos, E. Romero, and A. Tomas, SLEPc users manual, Tech. Report No. DSIC-II/24/02-Revision 3.7, D. Sistemes Informàtics i Computació, Universitat Politècnica de València, 2016.

[40] S. Balay, W. D. Gropp, L. C. McInnes, and B. F. Smith, in Modern Software Tools in Scientific Computing, edited by E. Arge, A. M. Bruaset, and H. P. Langtangen (Birkhäuser Press, Basel, Switzerland, 1997), pp. 163-202.

[41] Y. Gu and A. Kitaev, J. High Energy Phys. 02 (2019) 075. 
[42] A. Romero-Bermúdez, K. Schalm, and V. Scopelliti, J. High Energy Phys. 07 (2019) 107.

[43] D. Bagrets, A. Altland, and A. Kamenev, Nucl. Phys. B911, 191 (2016).

[44] Z. Yang, J. High Energy Phys. 05 (2019) 205.

[45] H. T. Lam, T. G. Mertens, G. J. Turiaci, and H. Verlinde, J. High Energy Phys. 11 (2018) 182.

[46] We note that the series expansion of $F(t)$ in terms of $e^{\lambda t} / N$ relies on the fact that the SYK model contains a single positive Lyapunov exponent [11-13]. For a chaotic system with multiple Lyapunov exponents, $F(t)$ would have additional terms corresponding to each exponent, though at late times the terms with the largest Lyapunov exponent would remain dominant.

[47] Y. Saad, SIAM J. Numer. Anal. 29, 209 (1992).

[48] Our parallelized dynamics code is available open source as the package DYNAMITE: https://dynamite.readthedocs.io/, https://doi.org/10.5281/zenodo.3606826.

[49] S. Goldstein, J. L. Lebowitz, R. Tumulka, and N. Zanghì, Phys. Rev. Lett. 96, 050403 (2006).

[50] R. Steinigeweg, J. Gemmer, and W. Brenig, Phys. Rev. Lett. 112, 120601 (2014).

[51] D. J. Luitz and Y. B. Lev, Ann. Phys. (Amsterdam) 529, 1600350 (2017).

[52] A. Georges, O. Parcollet, and S. Sachdev, Phys. Rev. B 63, 134406 (2001).
[53] G. Gur-Ari, R. Mahajan, and A. Vaezi, J. High Energy Phys. 11 (2018) 070.

[54] A. M. García-García and J. J. M Verbaarschot, Phys. Rev. D 96, 066012 (2017).

[55] J. S. Cotler, G. Gur-Ari, M. Hanada, J. Polchinski, P. Saad, S. H. Shenker, D. Stanford, A. Streicher, and M. Tezuka, J. High Energy Phys. 05 (2017) 118.

[56] W. Fu and S. Sachdev, Phys. Rev. B 94, 035135 (2016).

[57] L. Erdős and D. Schröder, Math. Phys. Anal. Geom. 17, 441 (2014).

[58] M. Berkooz, M. Isachenkov, V. Narovlansky, and G. Torrents, J. High Energy Phys. 03 (2019) 079.

[59] A. Chew, A. Essin, and J. Alicea, Phys. Rev. B 96, 121119(R) (2017).

[60] I. Danshita, M. Hanada, and M. Tezuka, Prog. Theor. Exp. Phys. 2017, $083 \mathrm{I} 01$ (2017).

[61] P. Gao, D. L. Jafferis, and A. C. Wall, J. High Energy Phys. 12 (2017) 151.

[62] J. Maldacena, D. Stanford, and Z. Yang, Fortschr. Phys. 65, 1700034 (2017).

[63] A. Kruchkov, A. Patel, P. Kim, and S. Sachdev, Phys. Rev. B 101, 205148 (2020).

[64] A. Chen, R. Ilan, F. de Juan, D. I. Pikulin, and M. Franz, Phys. Rev. Lett. 121, 036403 (2018).

[65] A. Altland, D. Bagrets, and A. Kamenev, Phys. Rev. Lett. 123, 226801 (2019). 